\title{
Implementation of Interleave Division Multiple Access (IDMA) with Multiple Users in Wireless Communication System
}

\author{
Farheen Begum \\ Dept of Electronics and \\ Communication Engineering, \\ Godutai Engineering College \\ for Women Kalaburagi, Karnataka
}

\begin{abstract}
This paper provides a review on the IDMA (Interleave Division Multiple Access) technology in wireless communication system based on Interleaver. In this paper, IDMA technique is proposed in AWGN channel. IDMA is a multi- user scheme in which chip Interleavers are the only means of user separation. The receiver involves a chip-bychip iterative multi-user detection. The IDMA performance in terms of bit error rate, bandwidth efficiency and complexity reduction is discussed. However, we observe that during iterative process, the IDMA output performs well with ISI Cancellation when numbers of users increases. Indeed, the increase in the number of users requires the independent processing of MAI and ISI that is carried out in IDMA.
\end{abstract}

\section{Keywords}

Additive White Gaussian Noise (AWGN), Interleave Division Multiple Access (IDMA), ISI -Intersymbol Interference, Multiple Access Interference (MAI).

\section{INTRODUCTION}

From last several years, the broadband communication service in wireless grows rapidly. It gains extensive popularity in all over the world. Due to various parameters in wireless communication systems, it also performs many personal or organizational requirements. It include mobility and cost effectiveness that need the transmission of high rate data are highly reliable in order to fulfill the increasing services applications such as high quality audio recording, messaging services, and video chatting in next generation mobile system that is $4 \mathrm{G}$ generation. Existing wireless technologies reliably cannot support high rates of data, because of these technology fading become very sensitive.

For broadband wireless networks, the various multiple access technique (MA) has been proposed to support multi-service transmissions over the shared wireless link. In wireless communication system, the multiple access technique is one of the most efficient methods, particularly used in cellular network by mobile phone communication system. In recent that is many years back, the availability in wireless networks can be exceeded by the use of bandwidth. It has been studied that, various techniques are used to make the efficiency of bandwidth utilization; is better more users can be allotted in the cell. So that it can provide sufficient space within each cell. Previously existed multi- access techniques like FDMA, TDMA and CDMA are used in 1G/2G/3G systems are suitable for voice communication only but it is not suitable for burst data traffic and high data rate transmission which would be the dominant part in $4 \mathrm{G}$ system for traffic load. For high mobility, the data rate is up to 100 Mega bits per second
(Mbps) and for low mobility the data rate is up to 1 Giga bits per sec (Gbps). But the $3^{\text {rd }}$ generation systems allows the data rate of nearly 3.6 to $7.2 \mathrm{Mbps}$. usually if the systems fulfill all these requirements then it can be considered as fourth generation $(4 \mathrm{G})$ systems.

There are different types of multiple approaching techniques which are proposed for $4 \mathrm{G}$ systems follows CDMA, MCCDMA, OFDMA and IDMA. In code division multiple access, every user assigned a single coded sequence and it is used to encode the significance of information signal. The receiver knows the sequence of the user code. After reception, it converts or decodes the received signal and retrieves the sequence of data. Hence the spectrum of the coded sequence is selected to be larger than the information signal.

In Multi-carrier CDMA, it is also a multiple access technique which is used in orthogonal frequency division multiplexing based telecommunication system. It permits the system to hold multiple-users at identical time. Multicarrier CDMA system is highly complex in receiver and exceedingly necessary for changing the spreaded code at high data rates in transmitter which build the system inefficient.

One of the most multi-carrier techniques that are used in modulation system that transmits the signal through multiple carriers is nothing but orthogonal frequency division multiplexing (OFDM). These sub carriers are orthogonal to each other and they have different frequencies. On the other side, the orthogonal frequency division multiplexing is quickly detect or response the slight changes in carrier or offset frequency and phase noise than compared to single carrier systems. OFDM subcarriers result in the appearance of inter-carrier interference (ICI) and common phase error (CPE) due to loss of orthogonality in OFDM. To maintain the condition of orthogonality and to eliminate the loss of collision between the Interleavers in the channel . In OFDM, the cyclic prefix needs to be greater than the time delay increases in the channel.

A basic fundamental of Interleave division multiple access i.e. IDMA is differentiated by two users in Interleaver. A multiuser technique in which chip Interleaved are only means of separating the users that is nothing but IDMA. The iterative multi-user detection is done by receiver in chip-by-chip form. In this work, by combining the OFDM and IDMA, we propose a new method referred as a multi-user system in the mobile radio environment.

All users can transmit their information in same time at same frequency band in OFDM and IDMA method. By using Interleaving technique, the orthogonality can be obtained between the users. The choice of good Interleaver must 
demonstrate that the inter leavers are weekly correlated, do not require large memory or large bandwidth to communicate between transmitter and receiver and easy to generate.

\section{IDMA MECHANISM}

In wireless communication system, Interleaving can be referred as a technique which is commonly used to overcome noise in the channel such as error burst or fading. In Interleaving process, the input data bits reorder itself such that consecutive bits of data are exchanged and splitted among various blocks in a known pattern among them. At receiver, the Interleaved data is arranged back to original sequence of bits with the help of de-interleaver. As a result, introducing the correlated noise in transmission channel seems to be statistically independent at the receiver in interleaving and thus allocate better error correction.

In IDMA system, there exist several areas which are still open for the researchers. Many of them includes the optimum design of integral parts of IDMA communication system and hence future applications of IDMA mechanism in other areas including satellite communication, LAN networking, optical communications, power line communications, MIMO system and UWB technologies. In addition to this its horizon are still open for investigation about optimum modulation, channel coding, spreading, interleaving, and detection techniques.

The IDMA can be performed in terms of bit error rate and compare its complexity with an Inter-symbol interference cancellation technique for AWGN multipath channel. Thus, it promises a better performance that is compared with OFDM and IDMA when the existing information is completely evaluated. However we noticed that during iterative process the OFDM and IDMA out performs the IDMA with ISI Cancellation when numbers of users are increased. Indeed, increasing the number of users in MAI and ISI is carried out in IDMA requires independent processing.

\subsection{Interleavers in IDMA Scheme}

In [2], the Interleavers based on multi-access method has discussed earlier for large bandwidth efficiency, performance is improved and receiver complexity is low. This method depends on interleaving as the only mean to differentiate the signal from particular users. Then it is named as interleave division multiple access (IDMA). The user-specific Interleavers play a vital function in IDMA system. In case of turbo codes and decoding, the decorrelation between adjacent bit sequence is not possible. The correlation between the Interleavers should compute, the signals that get affected strongly from other user and the decoding process of specific user also get effected [1]. Better the Interleaver decorrelation, lower iterations are essential for detection purpose in multi-user detection (MUD) technique [4]. The decorrelation among the various user for example 16 users. Specific Interleavers provide a mean to decreases the multiple access interference (MAI) from other users and thus helps in the convergence of detection technique. various set of Interleavers are considered to be practical if it is generated easily, and not more than two Interleavers are collided in the set. The transmitter and receiver doesn't store or communicate maximum bits in order to agree with interleaving sequence. It might be demonstrated that defining the correlation between the Interleavers. It can be used to produce the collision criteria, where zero crosscorrelation implies that, it is not collided. In IDMA systems, transmission is required for transfer the matrix Interleaver. Whereas in receiver, it consist of spreaded data along with the interleaving pattern and is related to the users. So that larger the size of the Interleavers, more bandwidth are consumed during transmission, more the orthogonality is achieved among interleaver [1].

\subsection{Schematic diagram of IDMA Transmitter and Receiver}

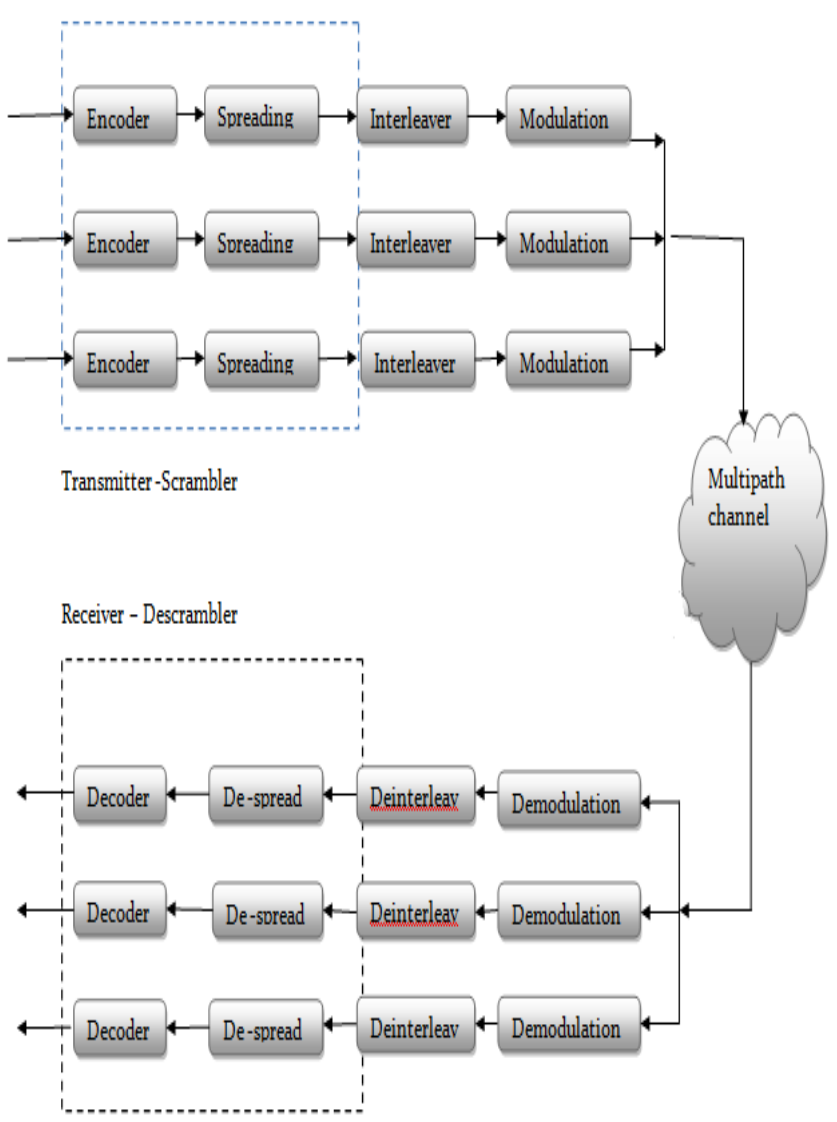

Fig 2.2: Schematic diagram of IDMA

The upper side of above figure shows transmitter the IDMA method. Users are slowly separated by Interleaver. The scrambler block is either identical or separate for different users . It can be the combination of both i.e., encoder and spreading. It is advantageous to use low-rate FEC code because it can provide extra coding gain from the performance point of view.

In IDMA system ,the basic ideas of Interleavers are opted for separating users must be orthogonal for all users and should be different for individual users. It is assumed that the generated Interleavers should be independent and random. Disperse the random Interleavers with coding sequence ,so that opposite chips are uncorrelated approximately and it facilitate the easy chip by chip detection method. Here, firstly we consider time variant single-path channels with real coefficients and BPSK signals.

Now the lower part of the figure 2.2, shows the receiver section of the IDMA system, iterative multi-user detector employs a chip by chip detection technique and avoid conventional MAP.

\section{CONVOLUTION ENCODER}

This applied to both continuous streams of data bits and to block of data. In convolution encoding, the information bits continuously runs through encoder likewise in block coding 
scheme where the information stream is first partitioned in to large number of blocks, then it is encoded.

\section{SPREADING}

Spreading the code is one of the major element in whole IDMA. IDMA codes are specific to each channel. When spreading codes are combat with data stream then transmitted data should gain the benefits of spreading .

\section{SCRAMBLER}

It is a device that transpose or invert the signal or else encodes the message bit at the transmitter side to make the message meaningless at the receiver. scrambler is a device that manipulates data stream before transmitting. It is also referred as a randomizer. Scrambling is often used for privacy.

\section{DESCRAMBLER}

The manipulation are reversed by a descrambler at the receiver end or descrambling used for decryption.

\section{INTERLEAVING}

It is a process of reshuffling the order of bits in one to one deterministic format.

\section{MODULATION}

This technique by which the signal wave is transferred from 0 to 1 and 1 to 0 in order to pass it over the communicating channel and reduce the noise effect is referred as modulation. This achieved by modulating the data by desirable modulation technique (BPSK).

\section{AWGN CHANNEL}

This is the channel through which the data is transformed. In Presence of noise,the signals get affected and cause distortion in its data content.

\section{DEMODULATING}

It is defined as a method where the receiver makes the complete use of carrier's phase to detect the signals. Then the receiver decides which of the feasible signal waveforms was transmitted from the output of the LPFs.

\section{DE-INTERLEAVING}

In communication system, The method of performing the deinterleaving mechanism having at least single Interleaver at the receiver. Deinterleaver is the reverse operation of interleaving. By using deinterleaving ,the security and accuracy of data transmission is increased.

\section{VITERBI DECODER}

In Viterbi decoding, When the sequence of data is received from the channel, it is essential to calculate original signal which is send already. The procedure of finding the original information sequence from the received data sequence can be done by a figure referred as trellis. Viterbi algorithm can be used for decoding the bit stream that is based on convolution code then it is encoded by using forward error correction.

\section{SIMULATION RESULTS}

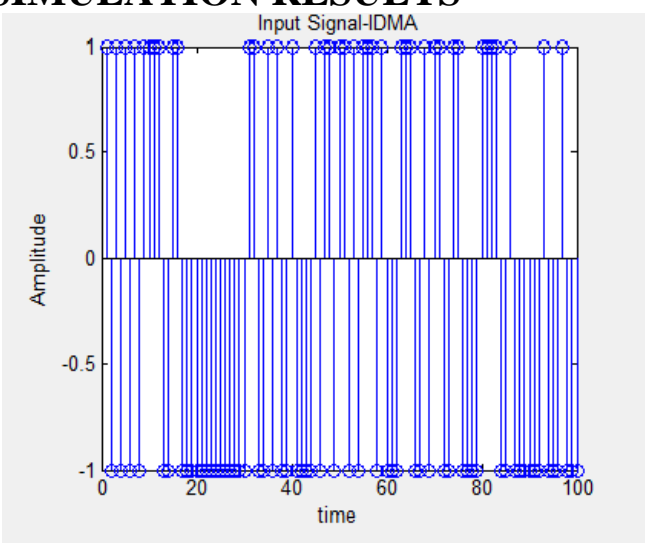

Fig 3.1 Input Signals.

The above figure 3.1 shows the input data 100 bits for IDMA.

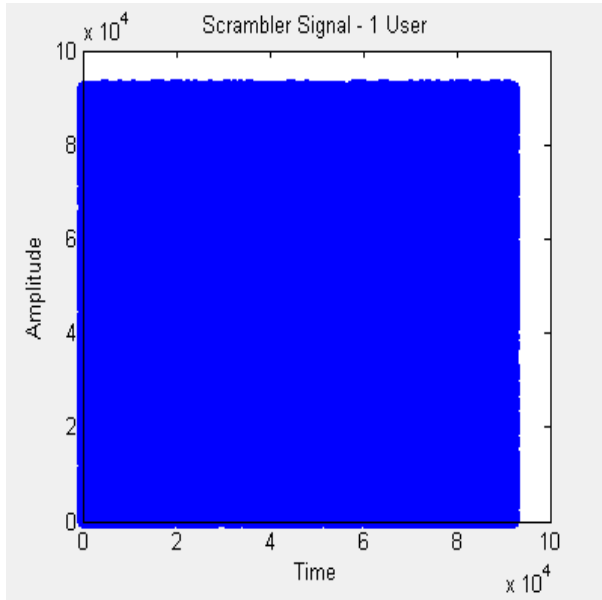

Fig 3.2 Scrambled Signal.

The above figure 3.2 shows the Scrambled signal (encoded \& spreaded) .with spreading length 90 bits. Scrambler is a device that manipulates data stream before transmitting. In convolution encoder, the message stream continously runs through the encoder .spreading codes are combined with the data stream that is to be transmitted .

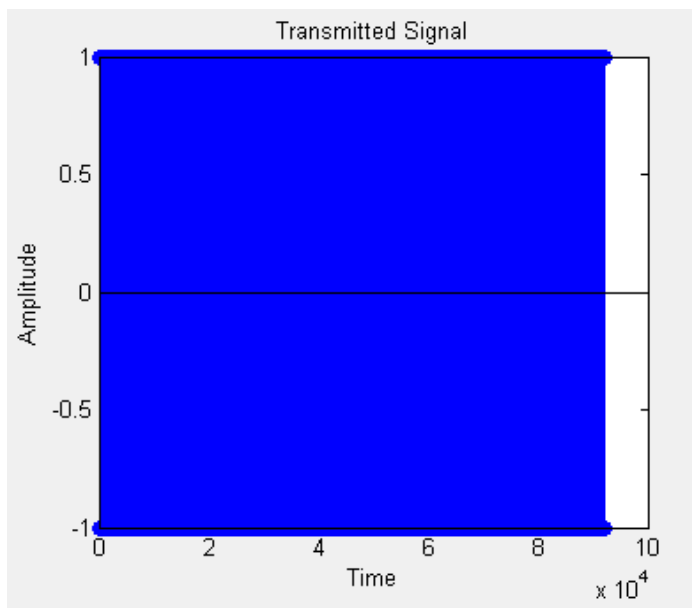

Fig 3.3 IDMA Transmitted signal.

The above figure 3.3 shows the transmitted signal with 90 bits, after encoding and spreading. 


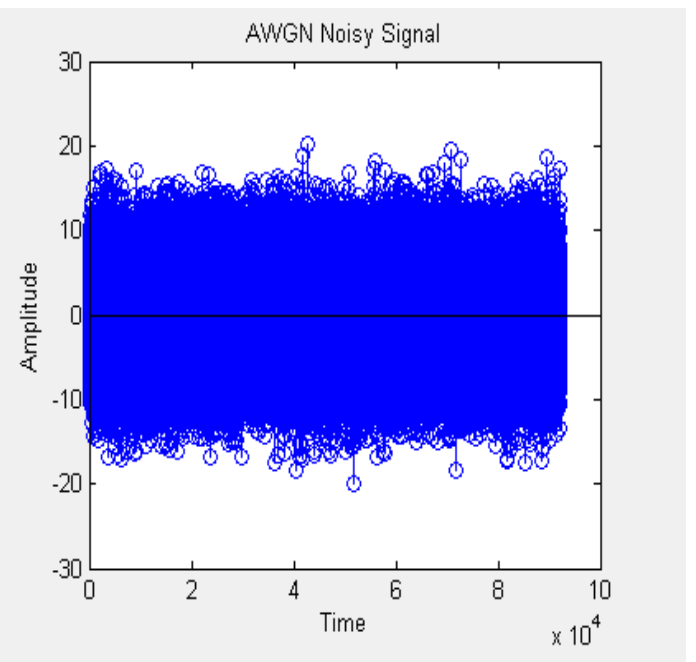

Fig 3.4 AWGN channel.

The above figure 3.4 shows the AWGN signal for IDMA. It is widely used to achieve reliable communication over a noisy channel and to make the error probability as small as desired.

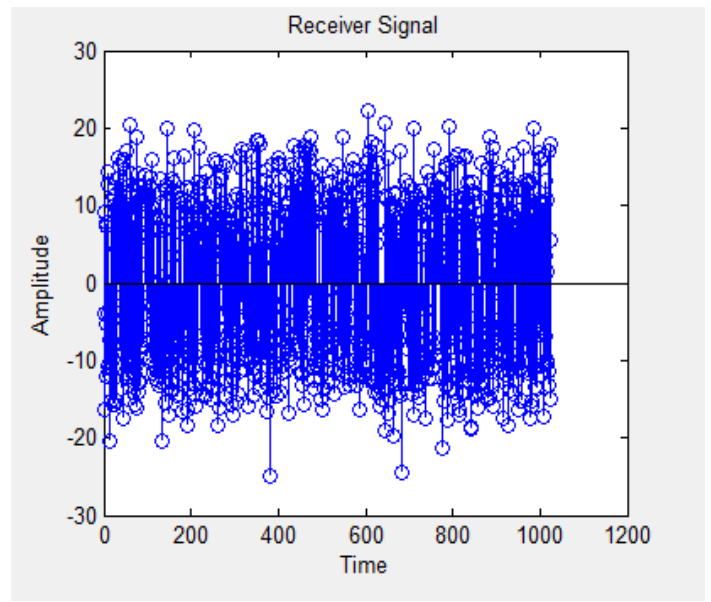

Fig 3.5 IDMA Received signal.

The above figure 3.5 shows the received signal with 90 bits. after demodulating and despreading .

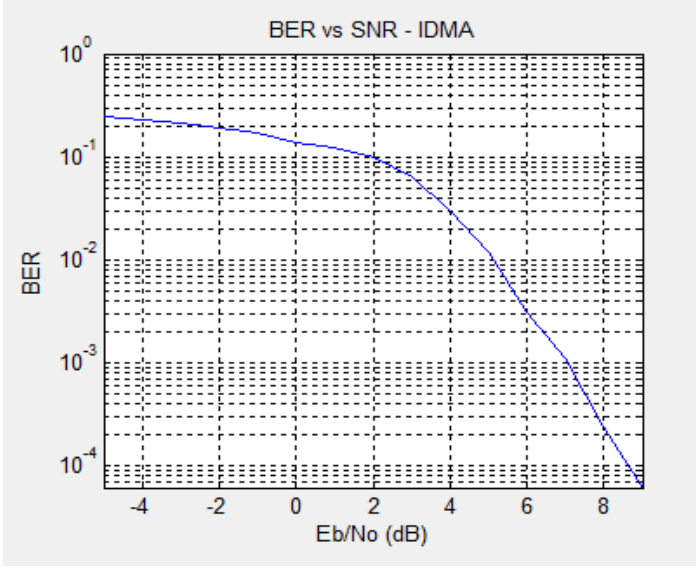

Fig 3.6 BER vs SNR.

The above figure 3.6 shows BER versus SNR graph for IDMA. Between -6 to $8 \mathrm{~dB}$ of SNR. BER is ranging from
0.31622 to 0.00025 . From 6 to $8 \mathrm{~dB}$ of SNR, BER ranging from 0.00158 to 0.00025 .

Table 1: Parameters for IDMA

\begin{tabular}{|c|c|}
\hline Parameters & Value \\
\hline Modulation & BPSK \\
\hline Noise & AWGN \\
\hline No. of bits & 90 \\
\hline Spreading length & 90 \\
\hline Data Length & 1024 \\
\hline No of users & 16 \\
\hline
\end{tabular}

\section{Graph For Multiple No Of Users BER vs No of users for IDMA}

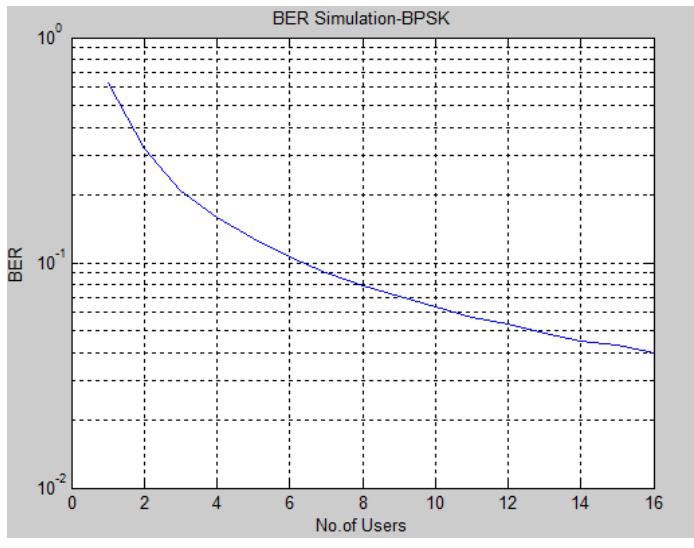

Fig 3.7 BER for IDMA.

The above figure 3.7 shows the BER simulation for IDMA. number of users 16. As the no of users increases the BER decreases in IDMA.

\section{CONCLUSION}

We have outlined the basic principles of IDMA and the simulation results with the help of AWGN channel. Based on the implementation of IDMA. MAI \&ISI can be overcome by the use of interleave division multiple access scheme i.e. IDMA. IDMA can mitigate interference among users to a maximum extent and provides high data rates without compromising the required quality of service. From the experimental results, the BER performance of IDMA is reduced. Hence we can conclude that the Computational complexity \& memory requirement is also reduced. So that , IDMA perform well for extra-large number of users. Thus it can be more suitable for future improvement in wireless communication systems. The proposed scheme works well for BER performance of IDMA compared to other existing techniques. Hence it is a new idea with many challenging issues in IDMA such as channel estimation, pilot assisted signalling design, adaptive transmission and resource allocation. Further data rate services can be increased; complexity can also be reduced using various efficient diversity techniques in IDMA for superior performance. IDMA can be used for various applications such as MIMO,MANET, ADHOC, 4G-UPLINK, UWB \&WLAN etc. 


\section{REFERENCES}

[1] L.Ping, L. Liu, K. Wu, and W.K. Leung, "InterleaveDivision Multiple-Access," IEEE Trans. Wireless Commun., Vol.5,No. 4, pp.938-947, Apr.2006.

[2] P.Wang, J. Xiao, and L. Ping, "Comparison of orthogonal and non-orthogonal approaches to future wireless cellular systems," IEEE Veh. Technol. Mag., vol.1, no. 3, pp.4-11, Sept.2006.

[3] Peter Hammarberg and Fredrik Rusek. "Channel Estimation algorithm for OFDM-IDMA: complexity and performance", IEEE Transactions on wireless communication, vol.-11, pp.1723-1734, no.5, may 2012.

[4] Ruchir Gupta, B.K. Kanauji a, R.C.S. Chauhan, M.ShuklaMember IEEE, "Prime Interleaver for Multiuser Iterative IDMA Systems" in International Conference on Computational Intelligence and Communication Network, DOI 10.1109/CICN.2010.119.

[5] B.Muquet and M.De.Couville, "Blind and Semi-Blind Channel identification methods using second order statistics for OFDM transmission" IEEE Transaction on signal processing, pp.2745-8, Mar.1993.

[6] H.liu and G.Xu, "A deterministic approach to blindestimation," IEEE signal processing letters, vol.1, no.12, pp.205-7, Dec.1994.

[7] M.Shukla, V.K.Srivastava, S.Tiwari "Interleave Division MultipleAccessforWirelessCommunication":ICONGEN COM-06

[8] Kuldeep choudhary, P.S Sharma "Interleavers for IDMA Technology: A Comparison Survey": IJARCCE Vol.1, Issue 2, Apr 2012.

[9] Aashish Shukla, DhirajPurwar, Dileep Kumar "Multiple Access Schemes for (4G) Communication: A Comparison Survey": ISDMISC (IJCA) 2011.

[10] Li Ping and Peng Wang, Xiaodong Wang "RecentProgress in Interleave-Division Multiple-Access (IDMA)", 